\title{
An illustrative review to understand and manage metal-induced artifacts in musculoskeletal MRI: a primer and updates
}

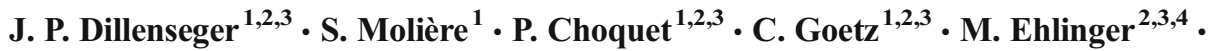 \\ G. Bierry $1,2,3$
}

Received: 7 August 2015 / Revised: 13 January 2016 / Accepted: 17 January 2016

(C) ISS 2016

\begin{abstract}
This article reviews and explains the basic physical principles of metal-induced MRI artifacts, describes simple ways to reduce them, and presents specific reduction solutions. Artifacts include signal loss, pile-up artifacts, geometric distortion, and failure of fat suppression. Their nature and origins are reviewed and explained though schematic representations that ease the understanding. Then, optimization of simple acquisition parameters is detailed. Lastly, dedicated sequences and options specifically developed to reduce metal artifacts (VAT, SEMAC, and MAVRIC) are explained.
\end{abstract}

Keywords MRI $\cdot$ Artifacts $\cdot$ Prosthesis

\section{Introduction}

Magnetic resonance imaging (MRI) studies the magnetic properties of tissues (proton density Rho, and relaxation times $\mathrm{T}_{1}$ and $\mathrm{T}_{2}$ ) by applying different calibrated magnetic fields: a static field $\mathrm{B}_{0}$, an alternative field $\mathrm{B}_{1}$, and three-directional gradient fields.

G. Bierry

guillaume.bierry@chru-strasbourg.fr

1 Medical imaging department, University Hospital of Strasbourg, 10 Av. Molière, F- 67098 Strasbourg, France

2 Icube, CNRS, University of Strasbourg, Strasbourg, France

3 Translational Medicine Research Federation, Strasbourg Medical School, University of Strasbourg, Strasbourg, France

4 Department of orthopedic surgery, University Hospital of Strasbourg, Strasbourg, France
These fields, used to generate and receive signals from the tissues, must be as homogenous as possible, and every field default will inexorably induce, causing spatial encoding mismatches and artifacts. Metal interferes massively with a magnetic field and induces local inhomogeneity that is responsible for characteristic artifacts, particularly troublesome in musculoskeletal imaging [1-3]. Metallic implants such as prosthesis or rods, due to their high magnetic susceptibility, impede correct spatial coding, leading to distortion, formation of areas of signal loss, and of areas of abnormal high signal ("pile-up" artifacts) [4].

Although these artifacts are easily recognizable (Fig. 1), their origins are more complex to handle, and are generally poorly understood by common users. To reduce these artifacts, basic strategies are available on all routine MR units and can be easily set up. Besides those tips, manufacturers continue to develop and propose specific reduction options.

In this review, we aimed to help radiologists to understand the physical mechanism of metal-induced artifacts, to adapt MR protocols in order to reduce artifacts in daily practice, and to present advanced reduction strategies developed by manufacturers.

\section{Physics of artifacts}

\section{Magnetic susceptibility}

Magnetic susceptibility is a dimensionless proportionality constant that indicates the degree of magnetization of a material (M) in response to an applied magnetic field (H). Magnetic susceptibility can be expressed in parts per million $\left(1 \mathrm{ppm}=10^{-6}\right)$. It is defined by the following relationship $[5,6]$ : 


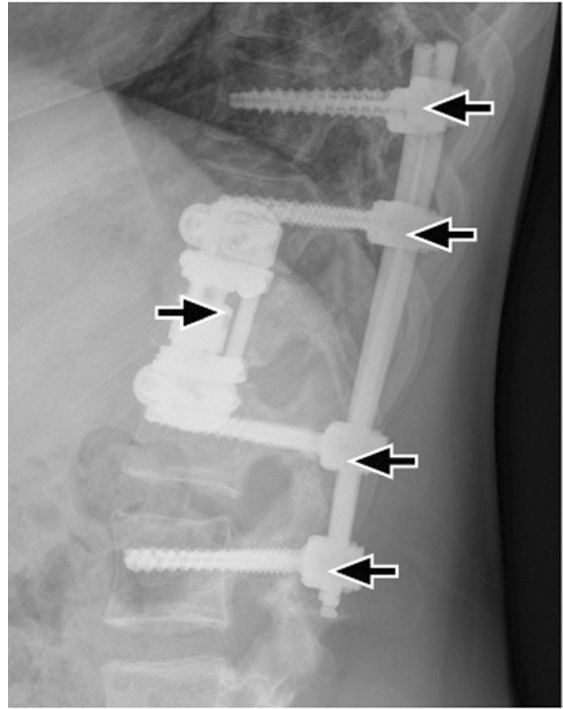

a.

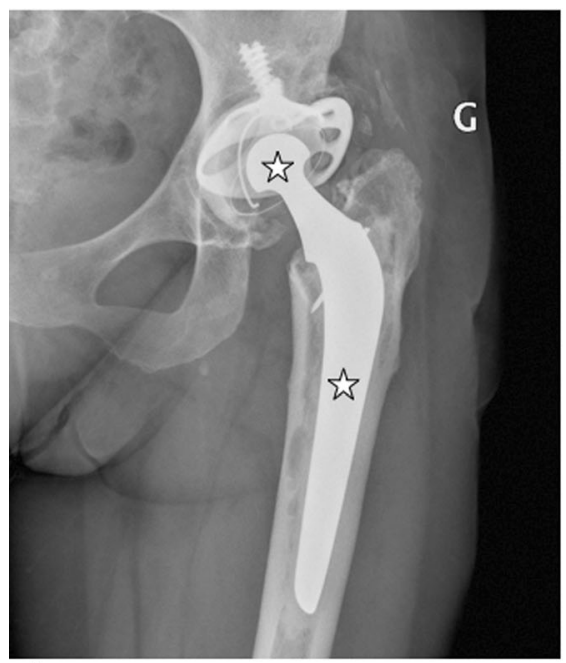

d.

Fig. 1 Metal-induced MR artifacts in spinal instrumentation and knee prosthesis. Spine instrumentation in a 50-year-old man: lateral radiograph shows intersomatic cage and rods with screws (arrows) (a) while sagittal T2-weighted fast spin-echo MR images (b and c) show artifacts. Total hip

$X=\frac{M}{H}$

$X$ is the magnetic susceptibility of the material (M)

$M$ is the magnetization of the material measured in amperes per meter $(\mathrm{A} / \mathrm{m})$

$H$ is the magnetic field strength measured in amperes per meter $(\mathrm{A} / \mathrm{m})$

Depending on the value of $X$, three magnetic effects can be distinguished: dia-, para-, and ferro-magnetism (Table 1) [5].

- Diamagnetism $(X<0 \mathrm{ppm})$ : is a property of all materials and always makes a weak contribution to the material's response to a magnetic field. For materials that show

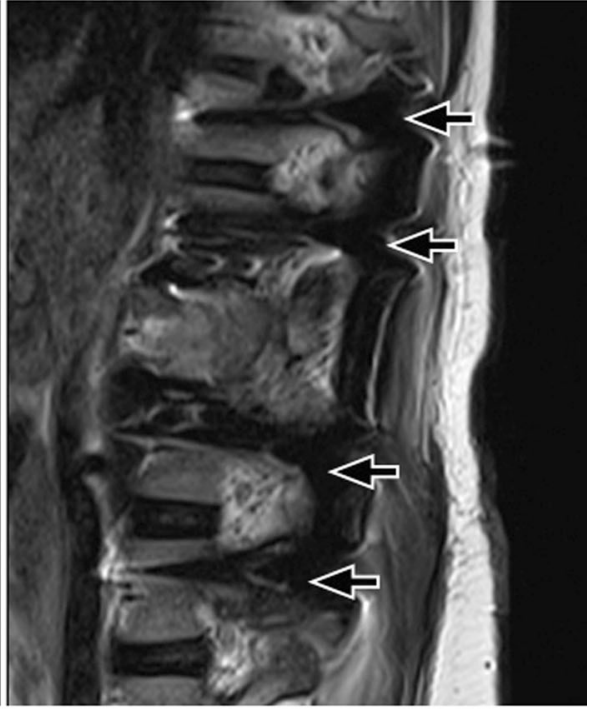

c.

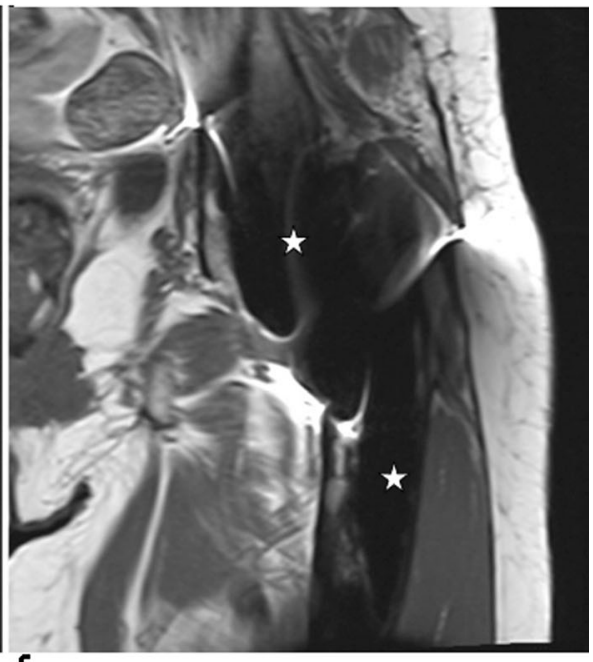

f.

replacement in a 62-year-old woman: hip radiography reveals head and shaft landmarks (stars) (d) while coronal T1-weighted fast spin-echo MR images (e and $\mathbf{f}$ ) demonstrate distortion and signal loss

some other form of magnetism (paramagnetism and ferromagnetism), the diamagnetic contribution may be regarded as negligible. Diamagnetic materials induce magnetic fields in the direction opposite to that of the applied magnetic field.

- Paramagnetism $(0<X<300 \mathrm{ppm})$ : some materials are attracted by an externally applied magnetic field and form internally induced magnetic fields in the direction of the applied magnetic field. Paramagnetic materials have a small, positive susceptibility to magnetic fields and are thus slightly attracted by a magnetic field. Paramagnetic properties are due to the presence of unpaired electrons and to the realignment of the electron paths caused by the external magnetic field. Unlike ferromagnets, 
Table 1 Magnetic susceptibility (in ppm) and type of magnetism of human tissues and materials

\begin{tabular}{lll}
\hline Material & $\begin{array}{l}\text { Magnetic susceptibility } \\
(\mathrm{ppm})\end{array}$ & Magnetism \\
\hline Water (soft tissue) & -9.05 & Diamagnetism \\
Cortical bone & -8.86 & \\
Liver & -8.76 & \\
Blood (deoxygenated) & -7.9 & \\
Liver (hemochromatosis) & 0 & \\
Air & 0.36 & \\
Aluminium & 20.7 & \\
Titanium & 182 & \\
Gadolinium contrast agent & 249 & Ferromagnetism \\
$\quad(0.1$ mol/l) & & \\
Chromium-cobalt & 900 &
\end{tabular}

paramagnets do not retain any magnetization in the absence of an externally applied magnetic field because thermal motion randomizes spin orientations.

- Ferromagnetism $(X>300 \mathrm{ppm})$ is the basic mechanism by which certain materials form permanent magnets, or are attracted to magnets. Paramagnetism and diamagnetism forces are usually so weak that they can only be detected by sensitive instruments in a laboratory, whereas ferromagnetism typically creates strong forces that are responsible of the common attraction phenomena of magnetism encountered in everyday life. Table 1 reports various tissues and materials according to magnetic susceptibility and type of magnetism.

\section{Artifact types and origins}

MRI is based on the recording of signals that are frequencyencoded by gradient fields applied in three orthogonal directions [4-10]. The application of a gradient in a homogeneous $\mathrm{B}_{0}$ field generates a linear variation in order to make spatial encoding achievable. Therefore, in a homogeneous magnetic field, the spatio-frequential relationship (the correspondence of spatial location and frequency encoding) is linear: to each spatial coordinate of the studied volume corresponds a single frequency coordinate. Metallic objects with high magnetic susceptibility placed within the magnetic field will generate spatial encoding errors that explain artifacts. Indeed, the spatio-frequential relationship then loses its linearity and a spatial encoding error offset takes place along the gradient's slope (Fig. 2) for protons exposed to higher frequencies in the center of the object. A signal accumulation appears in the offset front head (pile-up artifacts) (Fig. 2) associated with a loss of signal at the other extreme (Fig. 2) [4-7]. At the edges of the metallic object, the same encoding error offsets are observed, but in the opposite direction of the gradient slope, due to lower magnetic field: as presented in Fig. 3, the magnetic field is locally more intense at the north and south poles and lower at the extreme borders of the object. The coexistence of areas of signal accumulation (dark stars) and signal loss (white stars) explains the typical "boomerang" appearance. As slice selection similarly required the application of a selection gradient, the above detailed mechanisms explain both in plane and through slice artifacts (Fig. 4) [10].

\section{Basic optimization of sequence parameters}

\section{Read-out bandwidth increase}

One simple way to reduce in-plane artifacts is to increase the gradient read-out bandwidth: in our own experience, significant improvements are seen with a read-out bandwidth higher than $500 \mathrm{~Hz} /$ pixel: (Fig. 5a). Nevertheless, if this method seems obvious on a simple object such as a cylinder, the result is less predictable with metallic hardware such as prosthesis (Fig. 5b) [3, 4, 9-11]. As it can be seen in the relationship below (Eq. 2), the signal-to-noise ratio (SNR) is inversely proportional to the square root of the read-out bandwidth [12]. In this formula, the $\mathrm{K}$ factor includes hardwaredependent parameters such as coil, pre-amp and noise power spectrum, field strength-dependent factors, pulse sequencetype parameters, and tissue-dependent parameters (spin density, T1, T2). For 2D SNR where $\mathrm{K}$ is a constant, $\mathrm{FOV}_{\mathrm{x}}$ and $\mathrm{FOV}_{\mathrm{y}}$ are the field-of-view in $x$ and $y$ directions, $\mathrm{N}_{\mathrm{x}}$ and $\mathrm{N}_{\mathrm{y}}$ are the number of frequency and phase encoding steps, $\Delta_{\mathrm{z}}$ is the slice thickness, NEX is the number of signal averages, and BW is the read-out bandwidth.

$$
S N R=K \cdot\left(\frac{F O V_{X}}{N_{X}} \cdot \frac{F O V_{y}}{N_{y}} \cdot \Delta_{Z}\right) \sqrt{\frac{N_{X} \cdot N_{y} \cdot N E X}{B W}}
$$

\section{Slice thickness}

Through-slice artifacts can be reduced by increasing the slice selection bandwidth [5, 11, 13-16]. Consequently, this will reduce the number of excited protons and therefore the slice thickness, with decrease of the signal-to-noise ratio. To maintain a sufficient SNR, the use of thin slices will therefore require increasing the number of averages (NEX) with inherent longer examination time. A way to increase the slice selection bandwidth without impacting slice thickness would consist of enlarging the RF bandwidth, however, modification of the RF bandwidth is in general not user-accessible.

\section{Spin echo versus gradient echo sequences}

It is commonly though that gradient-echo sequences (GE) increase image distortion due to the presence of metal. In fact, 
Fig. 2 Origins of metal-induced MR artifacts. a The

spatio-frequential relationship (the correspondence of spatial location and frequency encoding) is linear in a homogeneous magnetic field. The relationship lost its linearity when a metallic object (limits on dotted yellow line) is present within the magnetic field: protons (in red color) exposed to non-expected frequencies in the center of the object present encoding error offset (gray arrow) along the gradient's slope direction. As a consequence, signal accumulates in the offset front head (= pile-up artifacts) (dark stars) associated with a loss of signal at the other extreme (white stars). b At the edges of the metallic object, the same encoding error offsets are created, but in the opposite direction of the gradient slope. Areas of signal accumulation (dark stars) and signal loss (white stars) explain the characteristic "boomerang" appearance
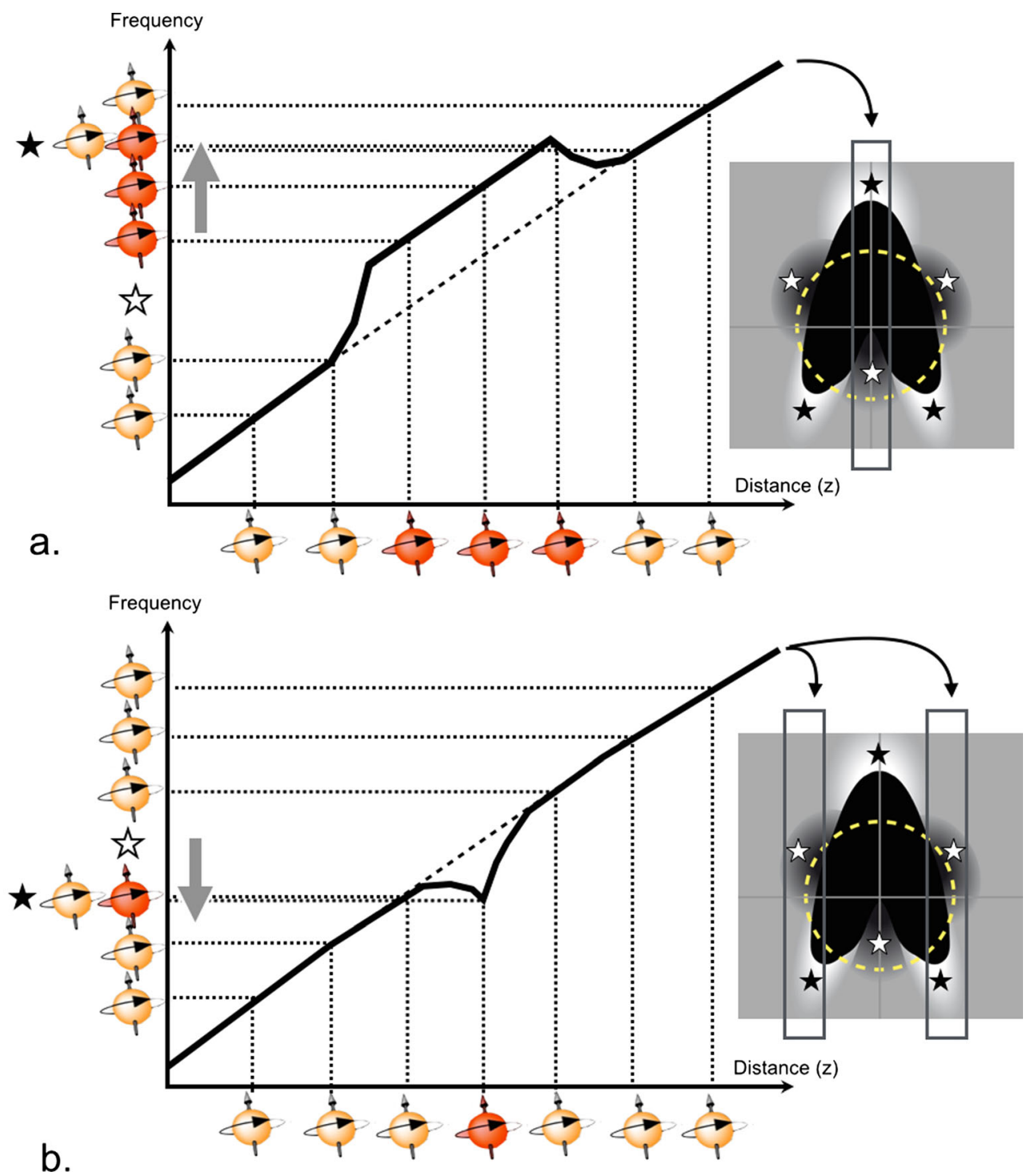

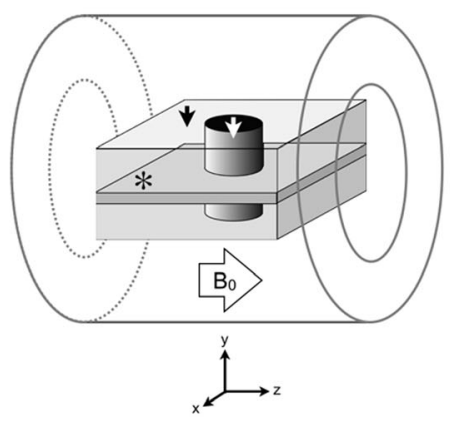

a.

Fig. 3 The "boomerang " aspect. a A cylinder (white arrow) fulfilled with gadolinium contrast agent at $0.1 \mathrm{~mol} / 1(249 \mathrm{ppm})$ is embedded in gelatinous water (black arrow) and placed in a MR field $\left(\mathrm{B}_{\mathbf{0}}\right)$. At this concentration, gadolinium provides no signal and simulates metalinduced artifacts. b Coronal (xz plane) spin-echo image of excited slice (* in a) shows in-plane artifacts, while dotted circlec represents the real limits of the xxcylinder, a typical "boomerang" appearance is seen with

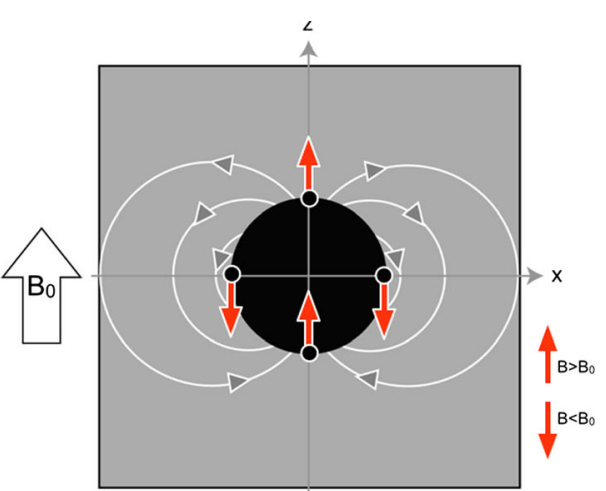

C.

pile-up hyperintensities localized in the sharpest bends (black stars) and lack of signal seen near the less pronounced curves of the boomerang (white stars). c Schematic representation of the magnetic dipole field outside the cylinder (grey arrowheads): the resulting forces (red arrows) are along $\mathrm{B}_{0}$ at the center, and opposite to $\mathrm{B}_{0}$ at the edge of the object, explain the boomerang shape 
Fig. 4 Experimental demonstration of in-plane and through-slice artifacts. a Experimental phantom containing a hip prosthesis $(\mathrm{CoCr} 29 \mathrm{Mo})$ immersed in gelatinous water $(8 \mathrm{~g}$ agar-agar $/ 1_{\text {water }}$ ). b Coronal T2w SE MR image through the hip prosthesis (1.5-T MRI system (Magnetom Aera, Siemens Medical Solutions, Erlangen, Germany; slice thickness $3 \mathrm{~mm}$ ) shows in-plane artifacts with pile-up (dark stars) and signal loss (white stars). c Sagittal reconstructions following dotted line show through-plane artifacts

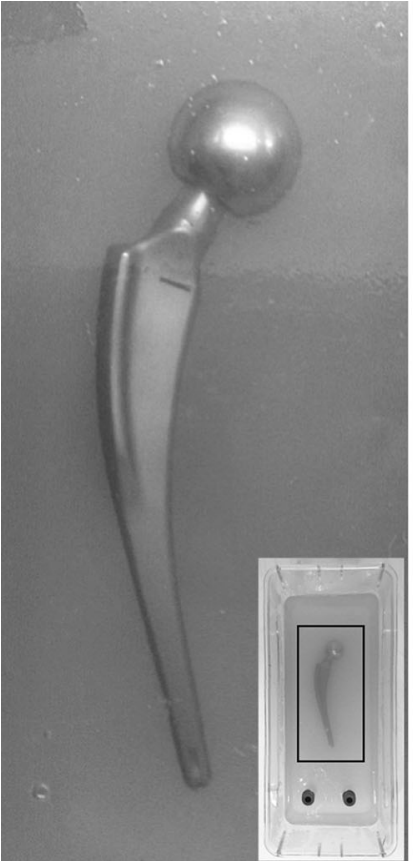

a.

the type of sequence does not impact directly image distortion, which is a consequence of the imaging mechanism. The differences between GE and SE sequences are faster spin-spin dephasing induced by the presence of metal: GE (under T2 * decay curve) are more sensitive to local field heterogeneity than spin-echoes (SE) (under a T2 decay curve). Thus, GE

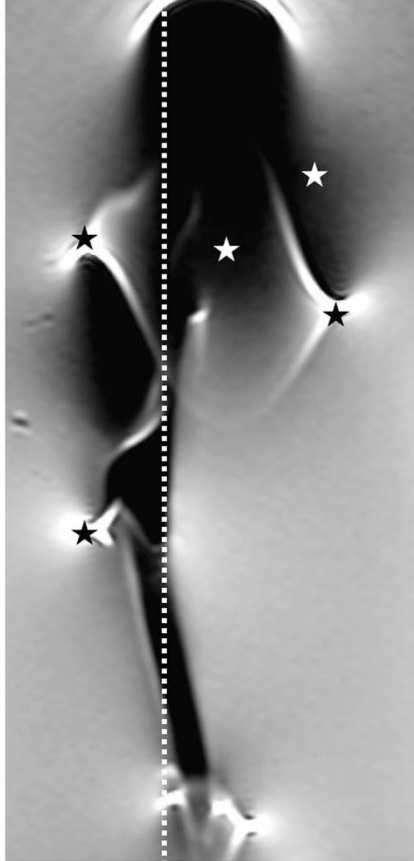

b.

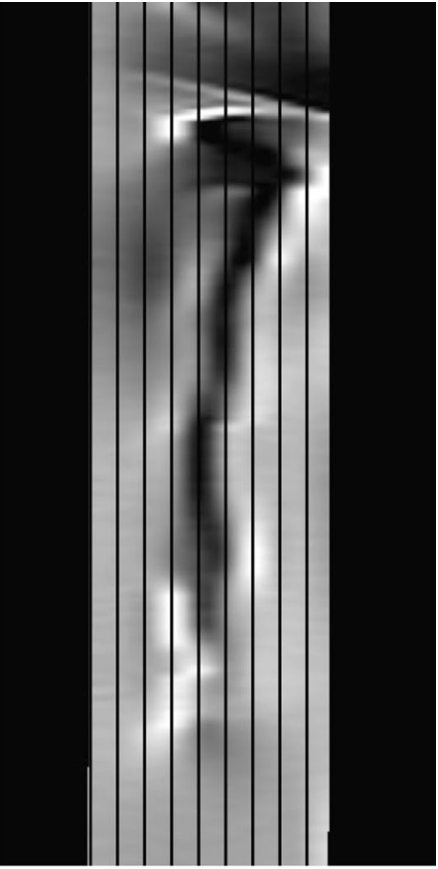

C.

sequences are very sensitive to field heterogeneity and generate a massive loss of signal, well beyond the limits of metalinduced distortions observed with SE sequences (Fig. 6). Prosthesis induces two kinds of artifacts: distortions (boomerang shape with signal pile-up and signal loss) and signal decrease due to premature spin-spin dephasing.
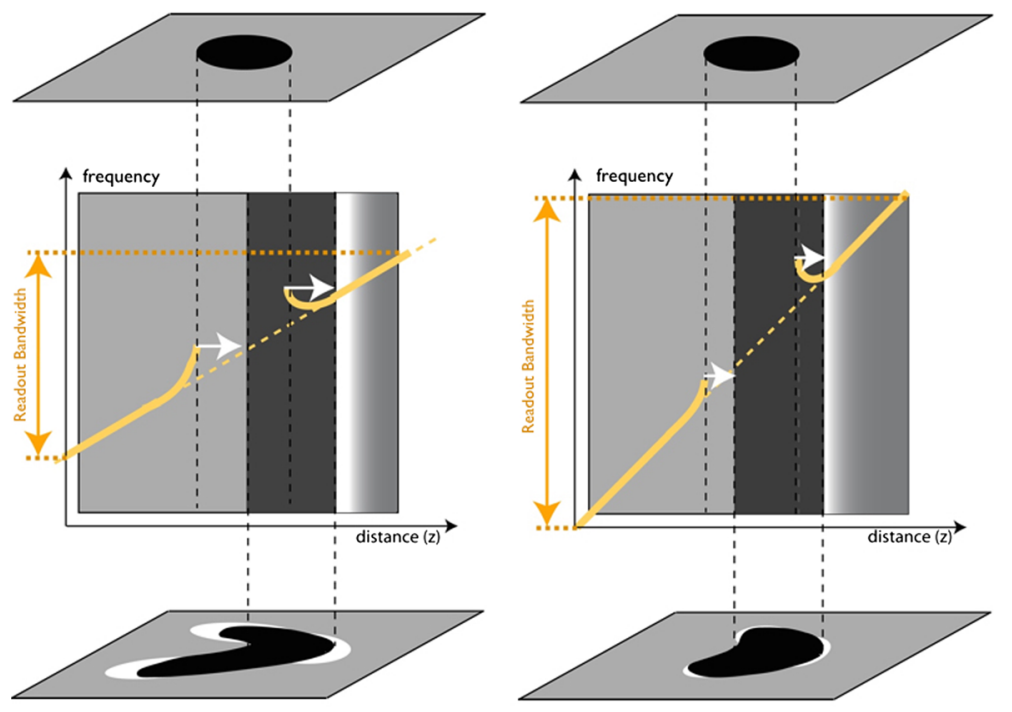

a.

Fig. 5 Bandwidth influence. a Schematic illustration of readout bandwidth increase: increasing bandwidth reduces signal misregistration (white arrow). b Corresponding bandwidth changes on

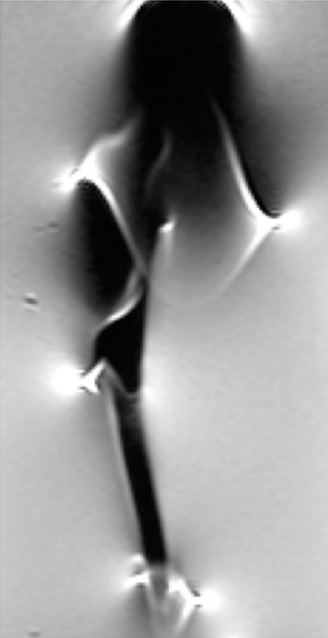

BW $150 \mathrm{~Hz} / \mathrm{pix}$

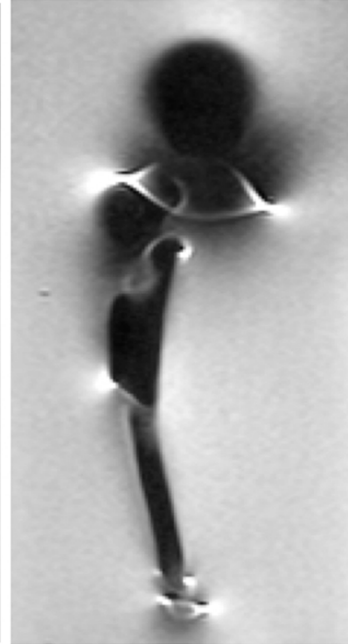

BW $450 \mathrm{~Hz} / \mathrm{pix}$ b.

CrCo prosthesis phantom: the "boomerang" appearance decreases as bandwidth increases ( $B W$ bandwidth) 
Fig. 6 Comparison of coronal T1w GRE image (a) and coronal T1w SE image (b) of a 50-yearold male patient with left total hip replacement $(\mathrm{Cr}-\mathrm{Co})$. Spatial distortions are present on the two images but with major signal decrease $(\mathrm{T} 2 *)$ on gradient-echo sequence
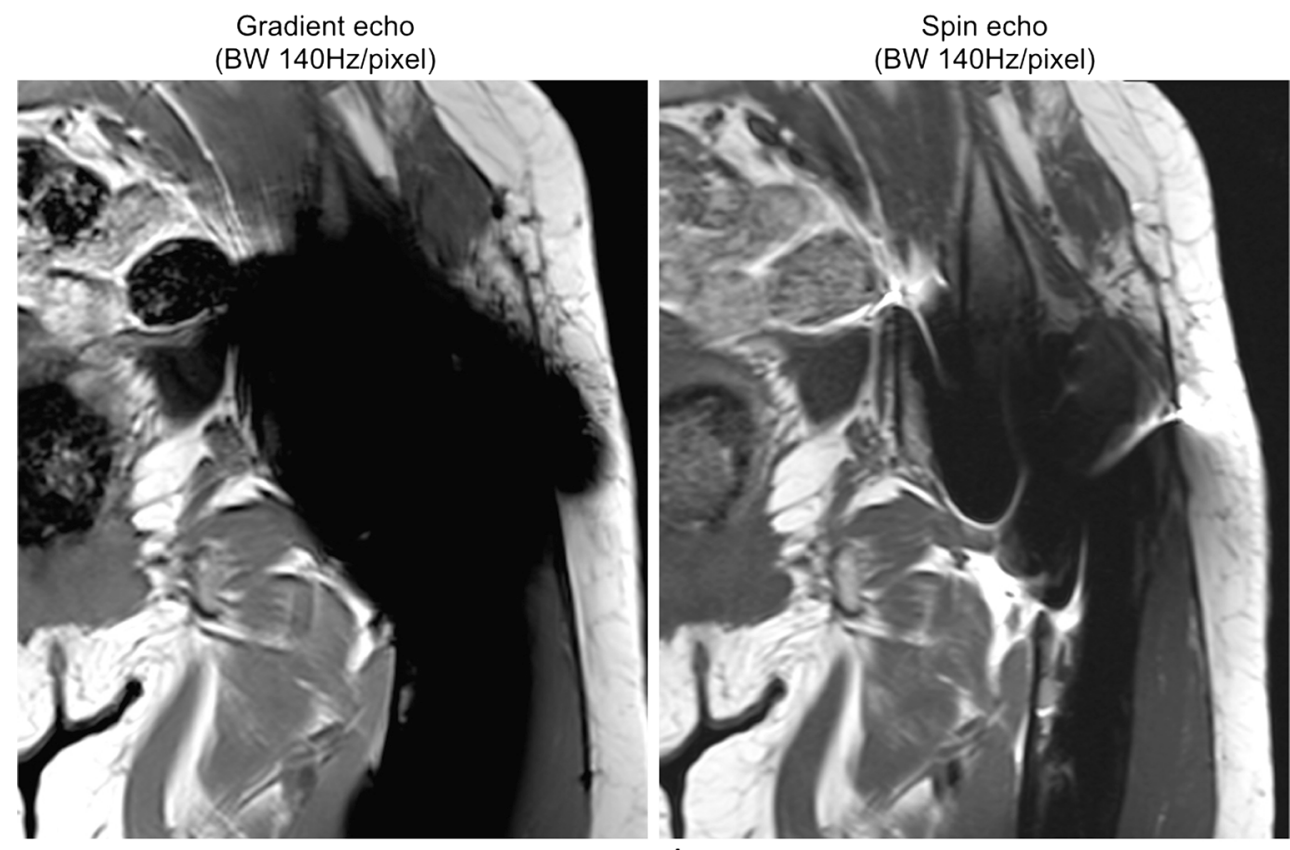

a.

b.
Therefore, SE sequences have to be preferred to GE when exploring metallic hardware. Moreover, fast SE techniques that employ long echo-train-length avoid premature signal decrease and are even more recommended $[6,13,15,16]$.

\section{Fat suppression}

There are, roughly speaking, two main groups of sequence to remove fat signal on MR. The first is based on the chemical shift phenomenon that occurs between protons $(\mathrm{H})$ of water and fat (208 Hz at 1.5 Tesla): pulse-selective saturation techniques (Fat Sat, Spir) and phase shifts techniques (water exCitation, ProSet, Dixon, etc.). Metal affects preferentially this group of sequences exploiting chemical shift (Fig. 7). Nevertheless, among them, multipoint sequences (Dixon, Ideal) provide better results than conventional fat-sat techniques $[6,15,17,18]$ (Fig. 8 ). Heterogeneity may be partially limiting, but not totally by applying a manual shimming.

The second group of techniques is based on inversion recovery, the most commonly used begin short tau inversion recovery (STIR). STIR is less sensitive to magnetic field inhomogeneity and provides a more homogeneous fat suppression than techniques based on chemical shift (Figs. 7 and 8). Unfortunately, due to short inversion time (150-200 ms at 1.5 T), STIR only allows T2 (long TE) or Rho (short TE)weighted contrasts $[18,19]$.

\section{Influence of magnetic field and metal materials}

Any non-metallic object placed inside the magnet induces a low variation of $\mathrm{B}_{0}$ due to diamagnetic (tissues) or paramagnetic properties (Table 1). This moderate increase in heterogeneity, on the order of $100 \mathrm{~Hz}$, which can be compensated with electromagnetic shim gradient but metal causes heterogeneity of several tens of $\mathrm{kHz}$ that cannot be fully compensated with shimming. Moreover, the stronger the magnetic field, the higher the magnetization of a metal object $[4,5$, 20-22] and the higher the magnetic field distortion will be. Increasing the magnetic field is therefore detrimental for prosthesis imaging: it is recommended to preferentially scan patients with metallic implants on a 1.5 -T rather than a 3T magnet.

The composition of the materials will directly impact the level of images distortion: metal-induced artifacts increase with the magnetic susceptibility of materials $[4,5,20-22]$ (Table 1). For instance, titanium implants (magnetic susceptibility of $182 \mathrm{ppm}$ ) generate less artifacts that chromium-cobalt (900 ppm) prostheses. In practice, users should know that it is easier to reduce metal artifacts from titanium materials than chromium-cobalt $[23,24]$.

\section{Dedicated MR sequences new developments}

Specific sequences are being developed to reduce metal artifacts; some of them are currently available at MR clinical units. The three main solutions offered by manufacturers are view angle tilting (VAT), multi-acquisition variable-resonance image combination (MAVRIC), and slice encoding metal artifact correction (SEMAC). We only aimed to describe below the basic principles of these methods; complete technical reviews are available in the literature and are beyond the scope of this article. 
Fig. 7 Experimental comparison of conventional (frequency

selective) fat saturation and STIR techniques. Coronal fat-saturation (a) and STIR (b) MR images of a phantom composed of a femoral metallic shaft $(*)$, a specimen of pure gadolinium chelates (black arrow) and a sample of fat (white arrow). Frequency selective fat saturation leads to imperfect fat suppression, but maintains signalto-noise ratio (SNR), whereas STIR provides uniform fat suppression but decreases SNR.

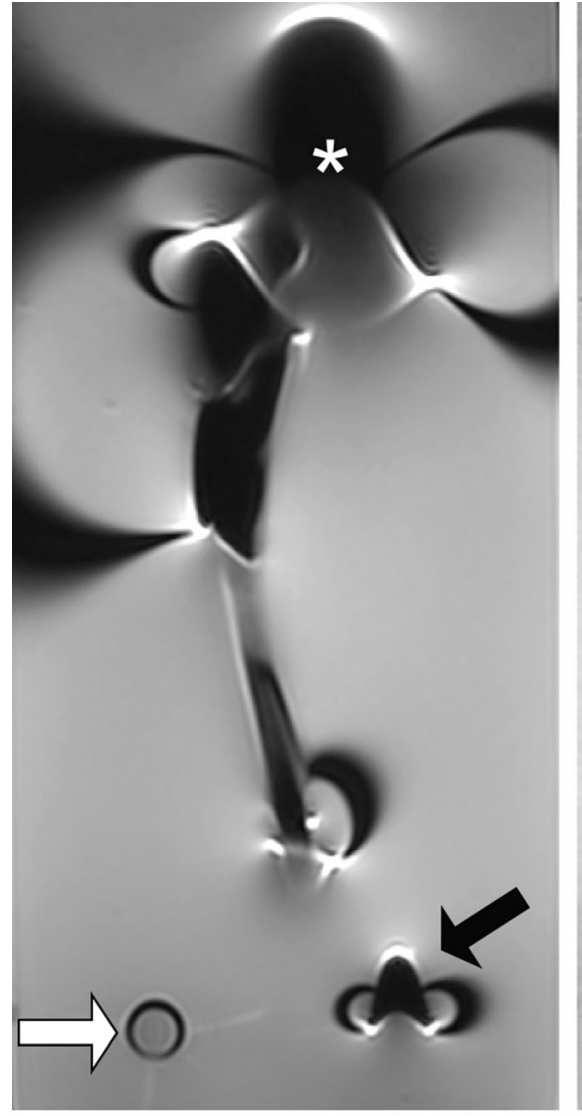

a.

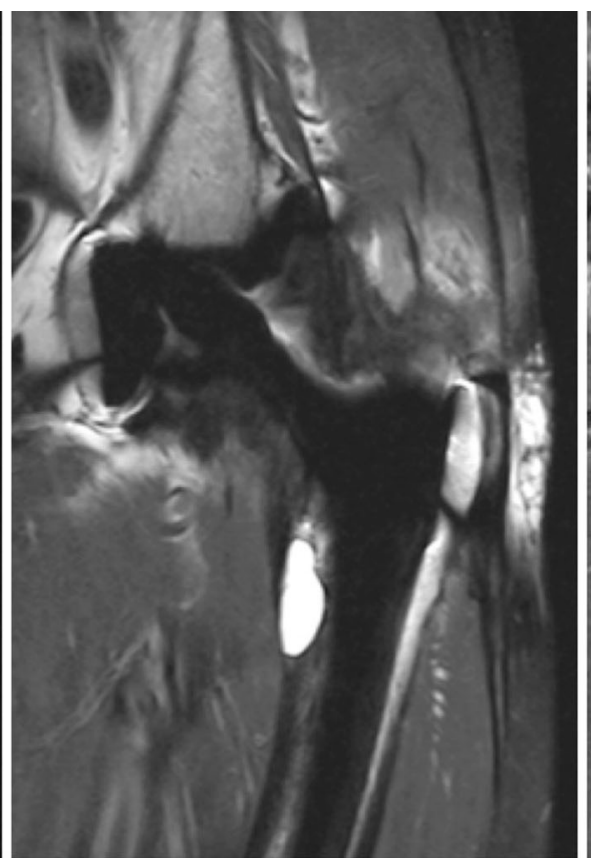

b.

a.

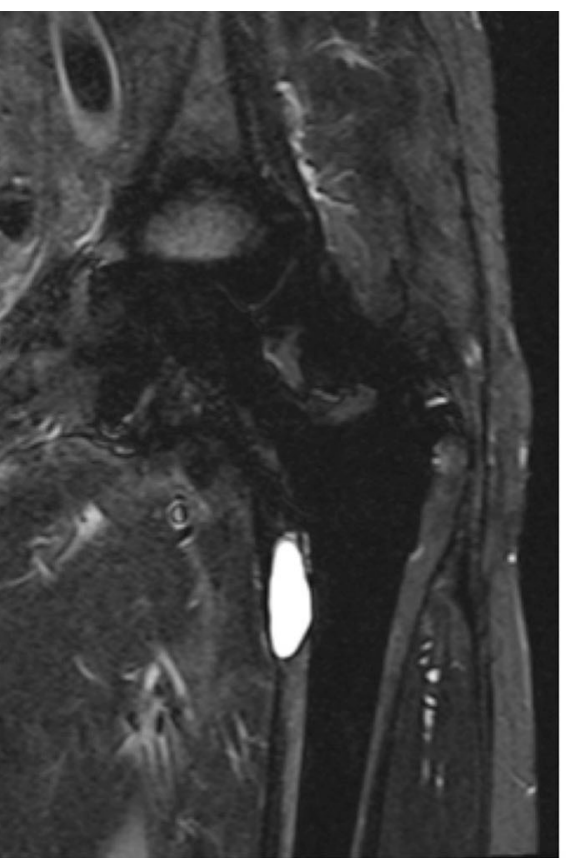

Fig. 8 In vivo comparison of fat suppression techniques coronal STIR (a), selective frequency fat-saturated T2w (b), and Dixon T2 (c) images of a 47-year-old female patient with left total hip replacement confirm the

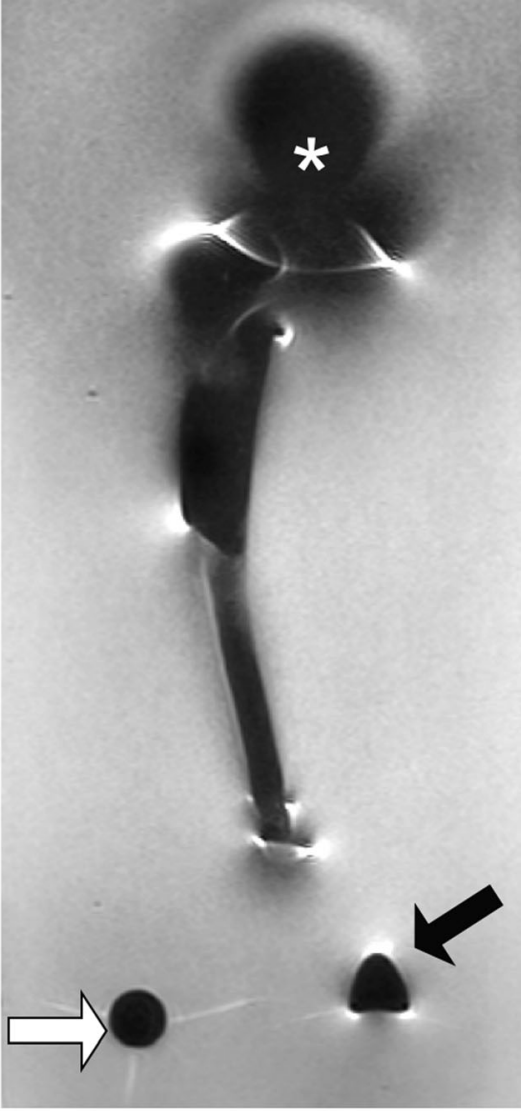

b.

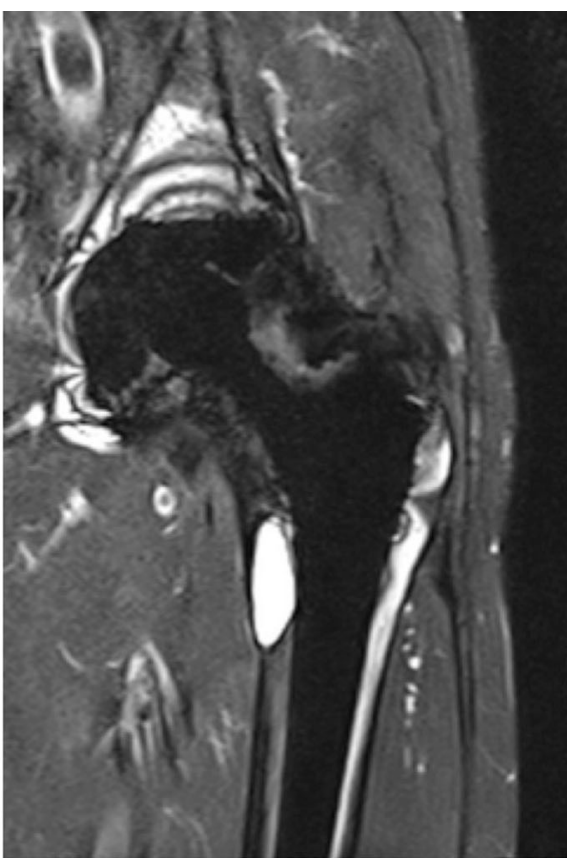

C.

more homogenous fat suppression of STIR sequence. Dixon T2 sequence is an interesting alternative, thanks to higher SNR 


\section{View angle tilting}

During the signal readout, a constant spatial encoding error exists for a given object, due to signal heterogeneity, and it can be estimated by a preliminary field mapping. The VAT technique applies, simultaneously, to the read-out gradient, a VAT gradient on the slice selection axis that tilts the signal sampling. The slice is effectively viewed at an angle [ $\tan -1(\mathrm{Gz} /$ Gx)]. The frequency shift due to field heterogeneity during readout is therefore compensated [23]. This option limits inplane distortions but remains ineffective in reducing throughplane artifacts. However, if VAT limits in-plane artifacts, it generates blurring on interfaces along the readout axis (Fig. 8) that can be reduce in implementing a large read-out gradient bandwidth (Fig. 9) [3][25-28].

\section{Multi-acquisition variable-resonance image combination}

When a metallic object is present within a magnetic field, protons of the same plane spin at different frequencies around the Larmor frequency (Fig. 10). This spectral dispersion of spins near metal implants is well beyond (150 ppm) levels typically encountered in magnetic resonance studies (3 ppm) and this inhomogeneity will distort the selected slab from its intended geometry. The MAVRIC technique uses a spectral approach: with judicious choices of RF spectral bands and increments of subimage frequency offsets, a complete composite image can be obtained via either maximum intensity projection or a sum of squares computation in the image domain (Fig. 10) [29]. The MAVRIC method successfully images in extreme off-resonance conditions by breaking abnormally broad spectral distributions into discrete and independently imaged frequency bins [29, 30]. However, acquisitiontime increases with the number of subimages used for the combination step. Currently, this method takes more than $10 \mathrm{~min}$, and can be shortened by optimizing the number of phase encoding steps and the RF pulse bandwidth.

\section{Slice encoding for metal artifact correction (SEMAC)}

The SEMAC technique aims to correct through-plane artifacts by sampling slice thickness with a phase encoding gradient. This allows protons location along the sliceselecting axis using phase coordinates (Fig. 11) [21, 31].
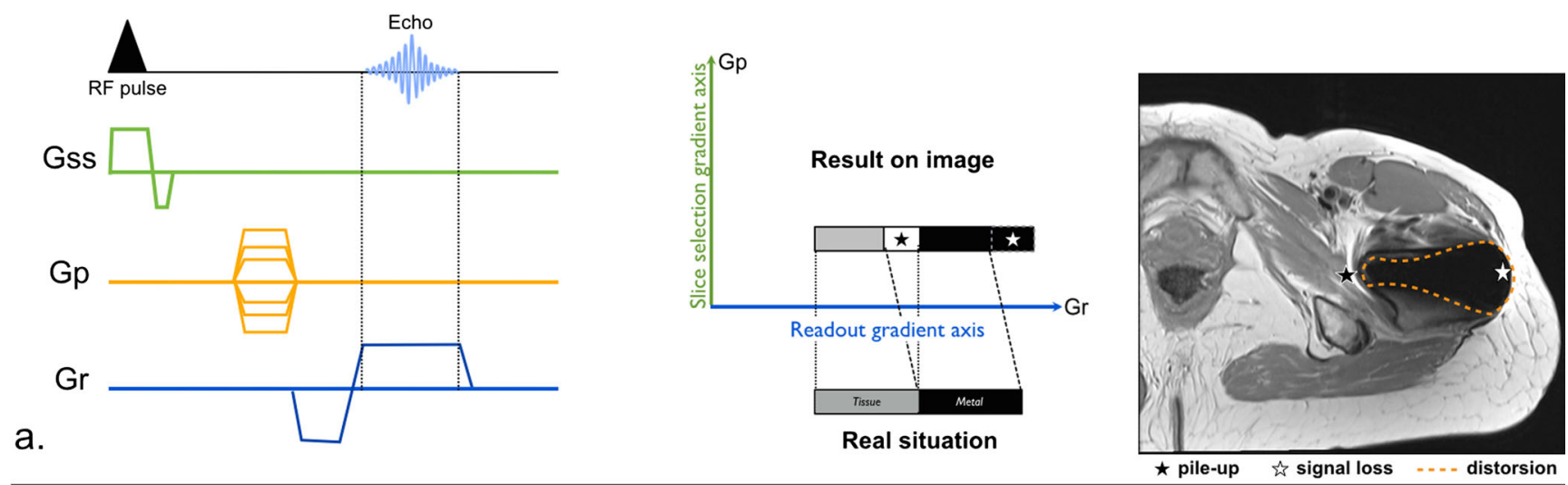
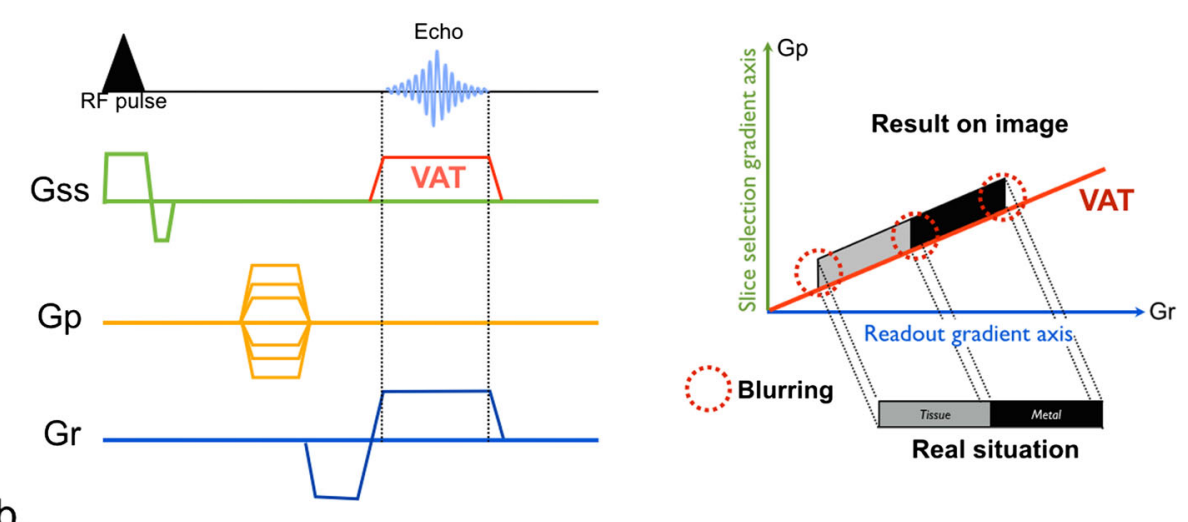

Real situation

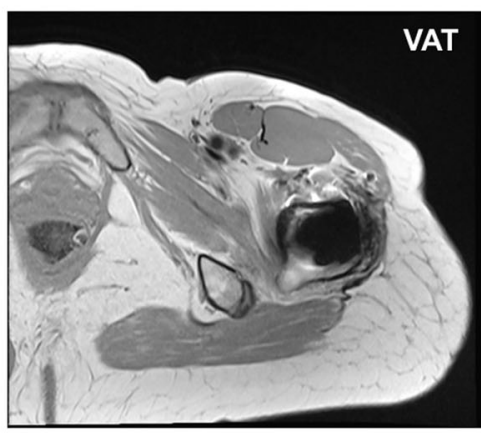

b.

Fig. 9 VAT sequence. a Conventional spin-echo sequence: coronal spinecho T1w MR image of a 57-year-old female patient with a total hip replacement $(\mathrm{Cr}-\mathrm{Co})$ shows in-plane artifacts. b VAT method applies a gradient on the slice-selecting axis during readout, with amplitude equal to that of the slice-selecting gradient. When viewed at this angle, shifts in the slice-selection plane compensate for shifts during readout, so that inplane shifts are registered again in the images 


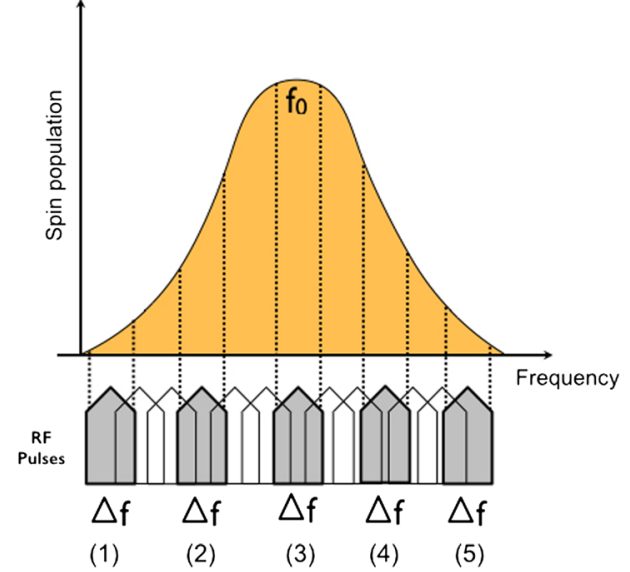

a.

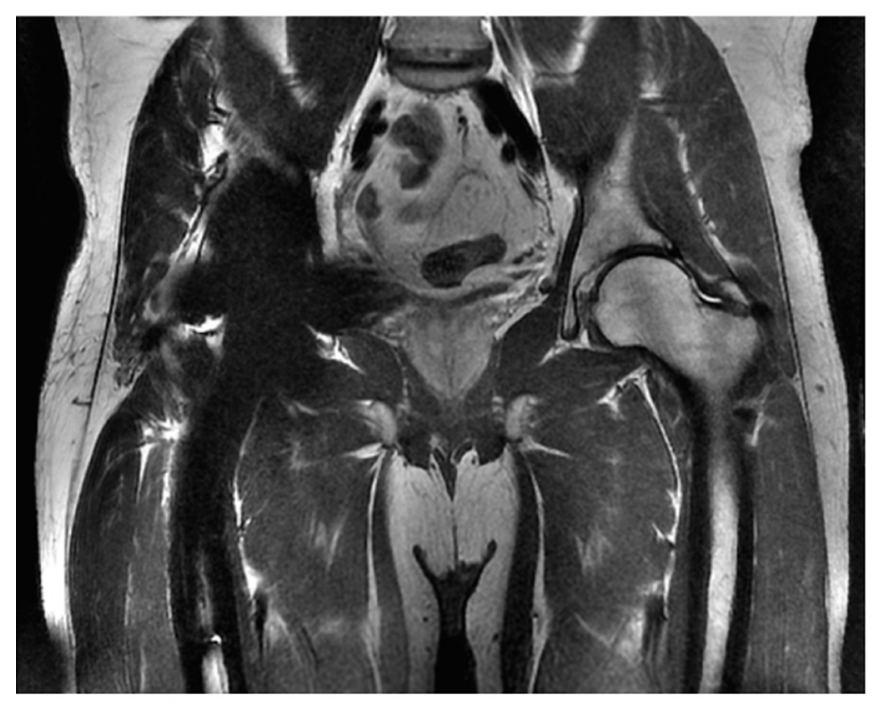

b.

Fig. 10 MAVRIC sequence. a With judicious choices of RF spectral bands and increments of subimage frequency offsets, a complete composite image can be obtained via either maximum intensity projection or a sum-of-squares computation in the image domain.

Protons with the same phase are combined to obtain a nondistorted slice. To further limit in-plane distortions, the SEMAC technique is in general combined with a VAT option [32-35] (Fig. 11). The use of this complementary phase encoding gradient increases acquisition times up to $10 \mathrm{~min}$.

\section{Perspectives}

The main trends are currently to optimize the above-presented technique $[33,35]$ notably in the hope of achieving a better compromise between acquisition time and artifact reduction. Recently, a hybrid acquisition sequence has been developed by combining SEMAC and MAVRIC techniques ("MAVRIC SL") [36]. This sequence aims to reduce metal artifact even

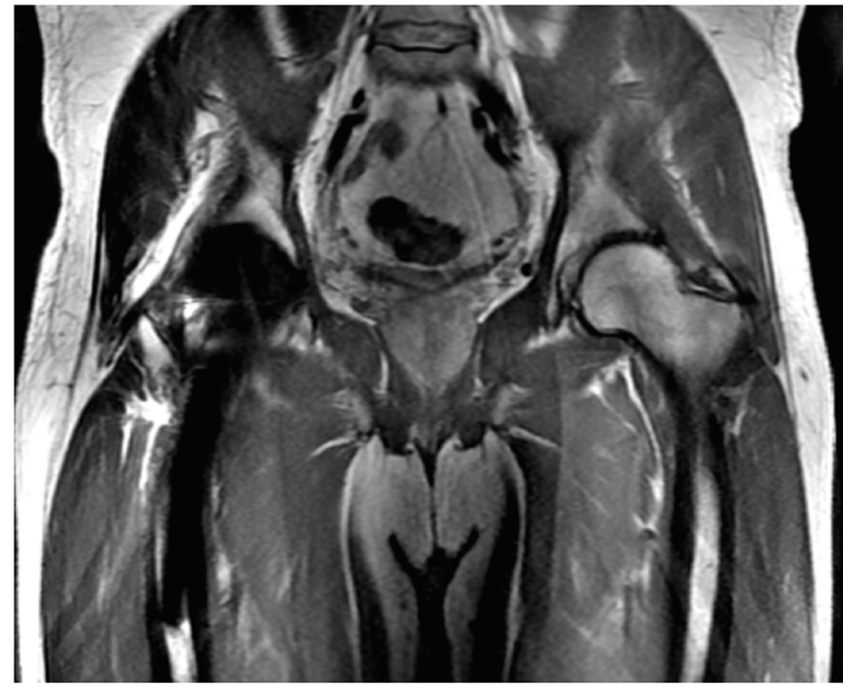

C.

Coronal proton density-weighted 3-T MR images of a 37-year-old male with a right hip without (b) and with (c) MAVRIC option where artifacts are notably reduced

with a field of higher strength than $3 \mathrm{~T}$ [37]. Compared with standard MRI, MAVRIC SL significantly improved the detection of clinically relevant findings, such as periprosthetic osteolysis [19, 37].

In order to avoid a premature loss of signal due to magnetic susceptibility, ultra-short echo-time sequences (UTE) could be interesting. These sequences were initially developed to study short-T2 tissues such as tendon, cortical bone, meniscus, and cartilage, but studies have highlighted their ability to reduce magnetic susceptibility artifacts [37]. Obtaining an instantaneous TE of about $0.1 \mathrm{~ms}$ is possible using a $\mathrm{k}$-space radialray filling. In this case, the free induction decay signal (FID) is directly placed in the center of the k-space. Combining this approach with MAVRIC allows the use of gradient echo sequences [38]. 

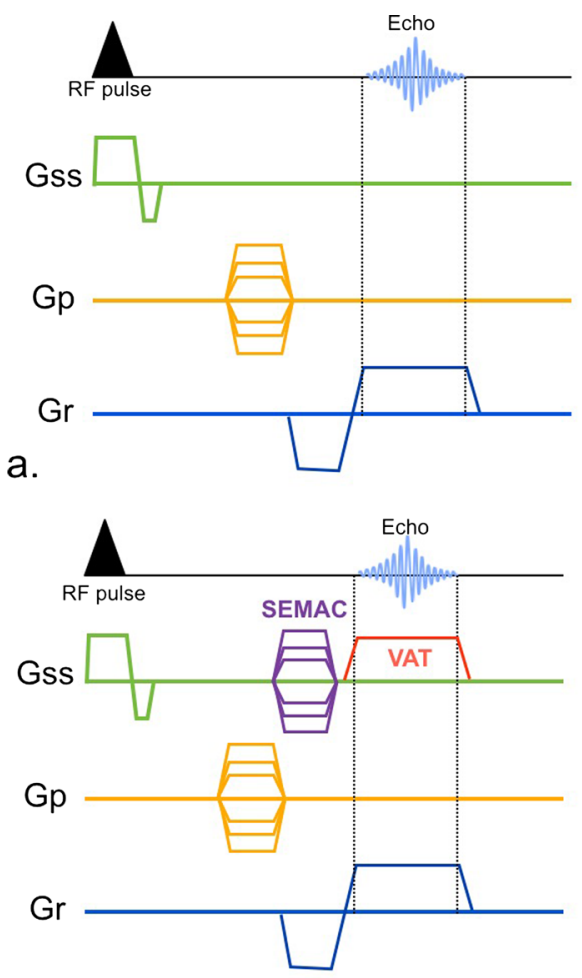

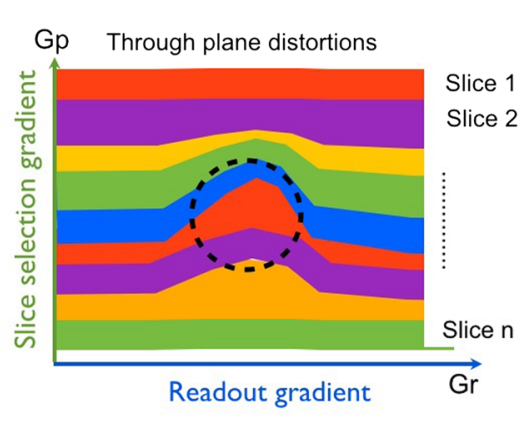

- - - metallic object

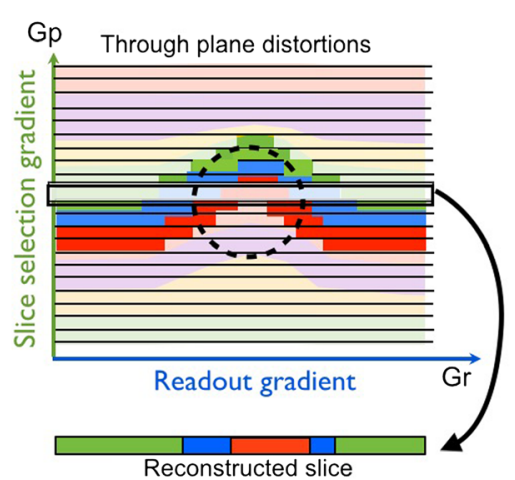

C.
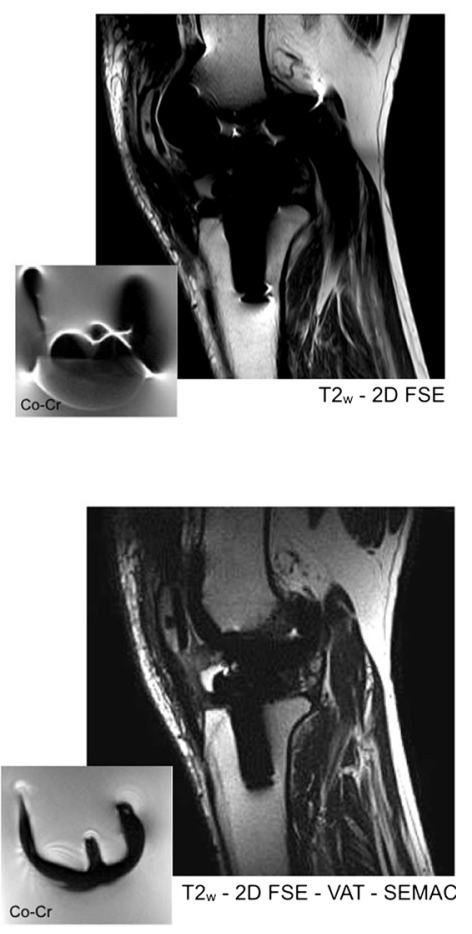

d.

profiles of encoded slices. Compared to standard FSE sequence (c), the VAT-SEMAC combination reduces both in-plane and trough-slice artifacts (d). (MR images with kind permission of Siemens Healthcare)

\section{Conclusions}

Understanding the occurrence of these artifacts remains essential for MRI users, both during sequence programming and interpretation steps. Metal-induced artifacts can be limited with simple solutions (Table 2), accessible to users (e.g., increasing read-out bandwidth, preferring STIR over fat-sat techniques, etc.), thanks to the recent availability of specific

Table 2 Common current tips and options to reduce metal-induced artifacts

\begin{tabular}{llll}
\hline Tips and options & Consequences & Limits \\
\hline 1 & Increase the readout bandwidth & $\begin{array}{c}\text { In-plane distortion (pile-up and signal loss) } \\
\text { reduction } \\
\text { Trough-slice distortion reduction } \\
\text { Reduce signal loss from dephasing }\end{array}$ & $\begin{array}{c}\text { SNR decrease }=>\text { acquisition time } \\
\text { increase (to reach SNR) }\end{array}$ \\
3 & $\begin{array}{l}\text { Reduce the slice thickness } \\
\text { Prefer SE, FSE rather than GE }\end{array}$ & Efficient and homogeneous fat suppression & $\begin{array}{c}\text { No access to dynamic contrast } \\
\text { enhancement techniques (rare) }\end{array}$ \\
SNR decrease
\end{tabular}


options and sequences [16]. Nowadays spine imaging with titanium implants is achievable without troublesome artifacts [23, 24, 28, 40-42], while MR imaging of chromium-cobalt prosthesis could remain challenging. Nevertheless, optimization of standard sequences as detailed above and availability of manufacturer-licensed dedicated techniques might help radiologists to overcome metal-induced artifacts in clinical practice $[13,14,17,19,38]$.

Acknowledgments The authors warmly thank Nadine Mischler and Franck Girard for illustrations and manuscript editing.

\section{Compliance with ethical standards}

Conflict of interest The author(s) declare that they have no competing interests.

\section{References}

1. Toms AP, Marshall TJ, Cahir J, et al. MRI of early symptomatic metal-on metal total hip arthroplasty: a retrospective review of radiological findings in 20 hips. Clin Radiol. 2008;63:49-58.

2. Cyteval C, Bourdon A. Imaging orthopedic implant infections. Diagn Interv Radiol. 2012;93:547-57.

3. Müller GM, Månsson S, Müller MF, von Schewelov T, Nittka M, Ekberg $\mathrm{O}$, et al. MR imaging with metal artifact-reducing sequences and gadolinium contrast agent in a case-control study of periprosthetic abnormalities in patients with metal-on-metal hip prostheses. Skeletal Radiol. 2014;43(8):1101-12.

4. Smith MR, Artz NS, Wiens C, Hernando D, Reeder SB. Characterizing the limits of MRI near metallic prostheses. Magn Reson Med. 2014. doi: 10.1002 /mrm. 25540

5. Schenck JF. The role of magnetic susceptibility in magnetic resonance imaging: MRI magnetic compatibility of the first and second kinds. Med Phys. 1996;23:815-50.

6. Lüdeke KM, Röschmann P, Tischler R. Susceptibility artefacts in NMR imaging. Magn Reson Imaging. 1985;3:329-43.

7. Hargreaves BA, Worters PW, Pauly KB, Pauly JM, Koch KM, Gold GE. Metal-induced artifacts in MRI. Am J Roentgenol. 2011;197:547-55.

8. Duerk JL. Principles of MR image formation and reconstruction. Magn Reson Imaging. 1999;7:629-59.

9. Paschal CB, Morris HD. K-space in the clinic. J Magn Reson Imaging. 2004;19:145-59.

10. Hopper TA, Vasilić B, Pope JM, et al. Experimental and computational analyses of the effects of slice distortion from a metallic sphere in an MRI phantom. Magn Reson Imaging. 2006;24: 1077-85.

11. Kolind SH, MacKay AL, Munk PL, Xiang QS. Quantitative evaluation of metal artifact reduction techniques. J Magn Reson Imaging. 2004;20:487-95.

12. McRobbie DW, Moore EA, Graves MJ, Prince MR. MRI from picture to proton, 2nd edn. Cambridge University Press, 2007.

13. Eustace S, Goldberg R, Williamson D, et al. MR imaging of soft tissues adjacent to orthopaedic hardware: techniques to minimize susceptibility artefact. Clin Radiol. 1997;52:589-94.

14. Toms AP, Smith-Bateman C, Malcolm PN, Cahir J, Graves M. Optimization of metal artefact reduction (MAR) sequences for MRI of total hip prostheses. Clin Radiol. 2010;65:447-52.
15. Aboelmagd SM, Malcolm PN, Toms AP. Magnetic resonance imaging of metal artifact reduction sequences in the assessment of metal-on-metal hip prostheses. Reports Med Imaging. 2014;7:6574.

16. Lee MJ, Kim S, Lee SA, Song HT, Huh YM, Kim DH, et al. Overcoming artifacts from metallic orthopedic implants at highfield-strength MR imaging and multi-detector CT 1. RadioGraphics. 2007;27(3):791-803.

17. Cha JG, Jin W, Lee MH, et al. Reducing metallic artifacts in postoperative spinal imaging: usefulness of IDEAL contrast-enhanced T1- and T2-weighted MR imaging - phantom and clinical studies. Radiology. 2011;259:885-93.

18. Bley TA, Wieben O, François CJ, Brittain JH, Reeder SB. Fat and water magnetic resonance imaging. J Magn Reson Imaging. 2010;31(1):4-18

19. Sutter R, Hodek R, Fucentese SF, Nittka M, Pfirrmann CW. Total knee arthroplasty MRI featuring slice-encoding for metal artifact correction: reduction of artifacts for STIR and proton densityweighted sequences. Am J Roentgenol. 2013;201(6):1315-24.

20. Arbogast-Ravier S, Gangi A, Choquet P, Brunot B, Constantinesco A. An in vitro study at low field for MR guidance of a biopsy needle. Magn Reson Imaging. 1995;13:321-4.

21. Farrelly C, Davarpanah A, Brennan SA, Sampson M, Eustace SJ. Imaging of soft tissues adjacent to orthopedic hardware: comparison of 3-T and 1.5-T MRI. Am J Roentgenol. 2010;194:60-4.

22. Farahani K, Sinha U, Sinha S, Chiu LC, Lufkin RB. Effect of field strength on susceptibility artifacts in magnetic resonance imaging. Comput Med Imaging Graph. 1990;14:409-13.

23. Suh JS, Jeong EK, Shin KH, Cho JH, Na JB, Kim DH, et al. Minimizing artifacts caused by metallic implants at MR imaging: experimental and clinical studies. AJR Am J Roentgenol. 1998;171(5):1207-13.

24. Stradiotti P, Curti A, Castellazzi G, Zerbi A. Metal-related artifacts in instrumented spine. Techniques for reducing artifacts in $\mathrm{CT}$ and MRI: state of the art. Eur Spine J. 2009;18(1):102-8.

25. Cho ZH, Kim DJ, Kim YK. Total inhomogeneity correction including chemical shifts and susceptibility by view angle tilting. Med Phys. 1988;15:7-11.

26. Butts K, Pauly JM, Gold GE. Reduction of blurring in view angle tilting MRI. Magn Reson Med. 2005;53:418-24.

27. Lazik A, Landgraeber S, Schulte P, Kraff O, Lauenstein TC, Theysohn JM. Usefulness of metal artifact reduction with WARP technique at 1.5 and $3 \mathrm{~T}$ MRI in imaging metal-on-metal hip resurfacings. Skeletal Radiol. 2015;44(7):941-51.

28. Griffin JF, Archambault NS, Mankin JM, Wall CR, Thompson JA, Padua JR, et al. Magnetic resonance imaging in cadaver dogs with metallic vertebral implants at 3 Tesla: evaluation of the WARPturbo spin echo sequence. Spine. 2013;38(24):E1548-53.

29. Koch KM, Lorbiecki JE, Hinks RS, King KF. A multispectral threedimensional acquisition technique for imaging near metal implants. Magn Reson Med. 2009;61:381-90.

30. Hayter CL, Koff MF, Shah P, Koch KM, Miller TT, Potter HG. MRI after arthroplasty: comparison of MAVRIC and conventional fast spin-echo techniques. Am J Roentgenol. 2011;197:W405-11.

31. Lee YH, Lim D, Kim E, Kim S, Song HT, Suh JS. Usefulness of slice encoding for metal artifact correction (SEMAC) for reducing metallic artifacts in 3-T MRI. Magn Reson Imaging. 2013;31:7036.

32. Hargreaves BA, Chen W, Lu W, et al. Accelerated slice encoding for metal artifact correction. J Magn Reson Imaging. 2010;31:98796.

33. Jungmann PM, Ganter C, Schaeffeler CJ, Bauer JS, Baum T, Meier $\mathrm{R}$, et al. View-angle tilting and slice-encoding metal artifact correction for artifact reduction in MRI: experimental sequence optimization for orthopaedic tumor endoprostheses and clinical application. PLoS One. 2015. doi:10.1371/journal.pone.0124922. 
34. Sutter R, Ulbrich EJ, Jellus V, Nittka M, Pfirrmann CW. Reduction of metal artifacts in patients with total hip arthroplasty with sliceencoding metal artifact correction and view-angle tilting MR imaging. Radiology. 2012;265(1):204-14.

35. Ai T, Padua A, Goerner F, Nittka M, et al. SEMAC-VAT and MSVAT-SPACE sequence strategies for metal artifact reduction in 1.5-T magnetic resonance imaging. Invest Radiol. 2012;47:267-76.

36. Koch KM, Brau AC, Chen W. Imaging near metal with a MAVRIC-SEMAC hybrid. Magn Reson Med. 2011;65:71-82.

37. Gutierrez LB, Do BH, Gold GE, Hargreaves BA, Koch KM, Worters PW, et al. MR imaging near metallic implants using MAVRIC SL: initial clinical experience at 3T. Acad Radiol. 2015;22(3):370-9.

38. Naraghi AM, White LM. Magnetic resonance imaging of joint replacements. Semin Musculoskel R. 2006;10:98-106.
39. Gatehouse PD, Bydder GM. Magnetic resonance imaging of short T2 components in tissue. Clin Radiol. 2003;58:1-19.

40. Petersilge CA, Lewin JS, Duerk JL, Yoo JU, Ghaneyem AJ. Optimizing imaging parameters for MR evaluation of the spine with titanium pedicle screws. AJR Am J Roentgenol. 1996;166(5):1213-8.

41. Chang SD, Lee MJ, Munk PL, Janzen DL, MacKay A, Xiang QS. MRI of spinal hardware: comparison of conventional T1-weighted sequence with a new metal artifact reduction sequence. Skeletal Radiol. 2001;30(4):213-8.

42. Song KD, Yoon YC, Park J. Reducing metallic artefacts in postoperative spinal imaging: slice encoding for metal artefact correction with dual-source parallel radiofrequency excitation MRI at 3.0 T. Brit J Radiol. 2013;86(1027):20120524. 